\title{
SEND Test System Common Name
}

National Cancer Institute

\section{Source}

National Cancer Institute. SEND Test System Common Name. NCI Thesaurus. Code C96433.

The common name for an animal used as the test system on a nonclinical study. 